\title{
Movimientos y desplazamientos en la investigación. Lo post-cualitativo como reconceptualización en dos tesis doctorales
}

\section{Movement and displacements in research. The post-qualitative seen as reconceptualization in two doctoral dissertations}

\author{
Sara Carrasco Segovia ${ }^{1}$ \\ sara_carrasco@ub.edu \\ Paola Villanueva González \\ paolavillanueva@ub.edu \\ Universidad de Barcelona, España
}

\section{Resumen:}

El presente artículo propone la perspectiva post-cualitativa como una oportunidad para pensar el sentido de investigar desde lugares no siempre legitimados; desde una mirada que nos mueve de nuestras zonas de estabilidad y que nos sitúa en una posición problemática que estimula el pensamiento y genera desplazamientos ontológicos, epistemológicos, metodológicos y éticos. Para ello, pretendemos revelar en la escritura de este texto cómo la perspectiva post-cualitativa nos ayudó a repensar y reconfigurar nuestros posicionamientos como investigadoras, profesoras y artistas a partir de nuestras tesis doctorales, así como los supuestos onto-epistemológicos y metodológicos que guiaban estas investigaciones. A partir de aquí, proponemos lo post-cualitativo como una perspectiva

\begin{abstract}
:
This paper presents the post-qualitative approach as an opportunity to rethink the meaning of research from positions which have not always been considered to be legitimate; from perspectives that move us from zones of stability to perspectives which place us within a problematic position, one that stimulates thought and generates ontological, epistemological, methodological and ethical displacements. For this purpose, we present the onto/epistemological and methodological assumptions which guide this research as well as how the adoption of a post-qualitative perspective helped us to rethink and reconfigure our positions as researchers, professors and artists during the developing processes of two doctoral theses. We look at the post-qualitative as a perspecti-
\end{abstract}

1 Dirección para correspondencia (correspondence address):

Sara Carrasco Segovia. Universidad de Barcelona. Departamento de Artes Visuales y Diseño. Unidad de Pedagogías Culturales. Facultad de Bellas Artes. Pau Gargallo, 4. 08028 Barcelona (España). 
que nos aporta una reconceptualización de las maneras de hacer y pensar la investigación y de cómo dar cuenta de ello. Una perspectiva que nos permite construir una investigación otra, reformular y repensar conceptos que han dominado la investigación cualitativa, y crear otros nuevos más allá de la tendencia a la domesticación que se ha hecho de ella.

\section{Palabras clave:}

Investigación; perspectiva post-cualitativa; reconceptualización de la investigación; desplazamientos onto-epistemológicos y metodológicos. ve in which we reconceptualize the ways of doing and thinking inquiry and how to explain it. A perspective that allows us to conduct a different kind of research, to reformulate and rethink notions of qualitative research, and create new concepts which aim to go beyond the trend of domestication that has taken place in social research.

\section{Key word:}

Research; post-qualitative perspectives; reconceptualization of research; onto/ epistemological and methodological shifts.

\section{Résumé :}

Cet article propose une perspective post-qualitative comme une occasion de réfléchir sur le sens de la recherche à partir de lieux qui ne sont pas toujours légitimés, depuis une perspective qui nous déplace de nos zones de conforts et qui nous met dans une position problématique qui stimule la pensée et génère des déplacements ontologiques, épistémologiques, méthodologiques et éthiques. À cette fin, nous avons I'intention de révéler dans la rédaction comment la perspective post-qualitative nous a aidés à repenser et reconfigurer nos positions en tant que chercheurs, professeurs et artistes à partir de nos thèses de doctorat, ainsi que les hypothèses épistémologiques et méthodologiques qui ont guidé ces recherches. Á partir de là, nous proposons l'approche post-qualitative comme perspective qui fournit une reconceptualisation des façons de faire et de penser la recherche et comment en rendre compte; une perspective qui nous permet de construire une recherche différente, de reformuler et de repenser les concepts qui ont dominé la recherche qualitative, et d'en créer de nouveaux au-delà de la tendance à la domestication qui en a été faite.

\section{Mots-clés :}

Recherche; perspective post-qualitative; reconceptualisation de la recherche; changements ontologiques; épistémologiques et méthodologiques.

Fecha de recepción: 22-11-2018

Fecha de aceptación: 24-5-2019 


\section{Introducción}

Entendemos la perspectiva post-cualitativa como una oportunidad para pensar el sentido de investigar, desde lugares no siempre legitimados; desde una mirada que nos mueve de nuestras zonas de estabilidad y nos pone en una posición problemática que estimula y genera desplazamientos ontológicos, epistemológicos, metodológicos y éticos. A partir de aquí, lo post-cualitativo nos aporta una reconceptualización de las maneras de hacer investigación y de cómo se da cuenta de ello (Jackson, 2013; Lather, 2013, 2016; Lather y St. Pierre, 2013; MacLure, 2013; Mazzei, 2013; St. Pierre, 2014, entre otras). Una perspectiva que nos permite construir una investigación otra; reformular y repensar conceptos que han dominado la investigación cualitativa (Deleuze y Guattari, 1983; Mazzei, 2013a, 2013b) y crear otros nuevos más allá de la tendencia a la domesticación que se ha hecho de ella.

Esto no solo nos lleva a repensar y reconfigurar nuestros posicionamientos como investigadoras que hemos finalizado hace poco tiempo nuestras tesis, sino también los supuestos ontológicos, epistemológicos y metodológicos que guían nuestras investigaciones. A partir de aquí, la pregunta de investigación no se fija como el lugar hacia donde queremos llegar -ese punto final al que supuestamente debe caminar una investigación-, sino como el lugar desde donde queremos partir sin miedo a perdernos. Nuestra intención ya no es la de reducir los datos, sino la de expandirlos. Pues la investigación no solo se fundamenta en la interpretación de unos datos como resultado de una tesis, sino en la revisión y reinterpretación constante del mismo recorrido de investigación. Esto produce un giro metodológico en la perspectiva de investigación que pone en cuestión el proceso de codificación y análisis de evidencias, así como su posterior visibilización en el producto final, cualquiera que sea su forma.

En relación con una de las investigaciones, titulada "Desplazamientos y tránsitos en un proceso de investigación sobre el lugar del cuerpo en las trayectorias y formación inicial de profesores de educación artística" (Carrasco, 2017), lo post-cualitativo actuó, por un lado, sobre el hacer y el pensar del proceso mismo de investigación; y, por otro, sobre el cómo se estaba pensando el cuerpo y cuál era su lugar en el proceso educativo de los/as profesores/as de artes visuales.

Inicialmente, la investigación perseguía respuestas sobre cómo un 
grupo de profesores de artes visuales en formación construían sus corporeidades y acciones performativas. Sin embargo, como resultado de los movimientos onto-epistemológicos y metodológicos experimentados, el foco de la investigación se movió hacia otros territorios expandiendo el significado del cuerpo y los diferentes lugares que éste ocupa dentro de las relaciones educativas; entendiéndolo no solo como una construcción social que depende únicamente de fuerzas humanas, sino como un territorio experiencial, de indecisión, indeterminación, conexiones, movimientos y relaciones (Barad, 2012; Grosz, 1994, 2004; RogowskaStangret, 2017). Es decir, una experiencia que atraviesa todo el espectro educativo donde lo material y lo espacial cobran gran importancia. De este modo, lo post-cualitativo actuó como reconceptualización del proceso de investigación y de su propósito inicial, pero también sobre la comprensión del cuerpo; trasladó el cuerpo a otro lugar, a un lugar inhumano, más que humano, más allá de lo humano, concentrando las zonas de proximidad entre el cuerpo y el mundo. Lo que Elizabeth Grosz (1987, 1994) denominó corpor(e)idad (corporeality).

En relación con la otra investigación, titulada "De qué hablamos cuando hablamos de Proyecto Artístico. Derivas desde los artistas, la institución y el arte contemporáneo" (Villanueva, 2017), lo post-cualitativo dibujó la forma de ser en la investigación, condicionando la relación con los sujetos implicados y el fluir de las conversaciones entabladas en las entrevistas, la manera de interpretar las experiencias que allí emergieron y la consecuente construcción de la narrativa resultante y presentada como la investigación. El sentido de "entrevista" y su esperado análisis, operó como un lugar donde "recoger" y "generar" teoría, así como acontecimientos pedagógicos en sí mismos (Atkinson, 2012) que pueden ser impredecibles, que están encarnados (Mazzei, 2013) y que se basan en perspectivas parciales (Haraway, 1988). La escritura, desligada así de un guion sólido, se convirtió en un lugar para perderse; en un ejercicio orgánico de hacer-deshacer y en el que dar cuenta de ello. Así, la historia pasó a ser un ensamblaje rizomático de experiencias corporeizadas, pretendidamente en devenir constante y a la deriva.

Desde estos movimientos, giros y perturbaciones, las dos investigaciones que aquí se presentan no se orientan a buscar resultados finales y concluyentes, sino a desvelar y problematizar el proceso a través del cual se han conformado con el propósito de abrir nuevos interrogantes para poder seguir pensando -y pensándonos como investigadoras, 
profesoras y artistas- dichas investigaciones. De ahí que estas tesis se configuren como ensayos del pensar y espacios de apertura a rutas imprevistas. Todo ello, desde unas formas de conocer que no eluden el no saber (Atkinson, 2010, 2012) y lo que nos perturba, donde tenemos en cuenta que la afectividad hace parte de nuestras investigaciones y que el aprendizaje real es siempre un proceso que nos afecta.

\section{La perspectiva post-cualitativa y sus aportes para pensar una investigación otra}

En esta ocasión no haremos una revisión y un tratamiento profundo de la investigación post-cualitativa y los fundamentos que la guían, algo que ya aparece en el primer artículo de este monográfico, sino que nos centraremos en reflexionar sobre su capacidad de reconceptualizar los procesos investigativos desde estas dos tesis doctorales, con el fin de: 1) visibilizar cómo lo post-cualitativo nos ha permitido pensar la investigación y el proceso de investigar desde otros lugares para generar nuevos conceptos sobre ello; 2) cómo lo post-cualitativo nos ha ayudado a expandir y no a reducir; y 3) cómo lo post-cualitativo nos ha permitido repensar y reconfigurar los supuestos ontológicos, epistemológicos, metodológicos y éticos que guían nuestras investigaciones.

Aunque el recorrido de esta perspectiva no se puede limitar solo a unas cuantas referencias, hemos realizado una selección de lecturas y autoras(es) que nos ayudan a repensar dichas cuestiones y sitúan los principales temas que han liderado el debate de la investigación postcualitativa. Esto, con el propósito de realizar un primer acercamiento que nos permita cartografiar los principales ejes que guían este artículo y esbozar el porqué de la necesidad de repensar y reconceptualizar nuestras investigaciones desde una perspectiva post-cualitativa.

Tal y como proponen Patti Lather y Elizabeth St. Pierre (2013) en su artículo Post-qualitative research, la tradición va siempre con nosotras y nosotras solemos perpetuar esa tradición en lo nuevo, provocando así una normalización de nuestros modos de hacer y de pensar la investigación en las ciencias sociales. "We always bring tradition with us into the new, and it is very difficult to think outside our training, which, in spite of our best efforts, normalizes our thinking and doing" (p. 630).

Esta tradición ha marcado largamente la investigación cualitativa (y 
cuantitativa) de supuestos positivistas y fenomenológicos acerca de la naturaleza y del mundo, privilegiando el saber sobre el ser y la lógica representacional y binaria (Lather y St. Pierre, 2013). De aquí que la perspectiva post-cualitativa nos ayude a cuestionar dichos límites para pensar en términos de simultaneidad, abandonar lo lineal y explorar diferentes patrones como enredos performáticos (entanglement).

En este sentido, la difracción como propuesta metodológica de Karen Barad (2003) -para pensar en los fenómenos no en términos de entidades preexistentes, sino como intra-acciones y fuerzas de las cuales otras cosas emergen-, conecta con la posibilidad de visualizar, desde la perspectiva post-cualitativa, las relaciones de una manera difractiva. Como propone Lisa Mazzei (2013b), se trata de deconstruir una trama de conexiones donde lo discursivo y lo material intra-actúan, se entienden y se constituyen mutuamente en la producción del conocimiento. Esto desafía la lógica representacional de la investigación, discutiendo el posicionamiento de la materialidad y lo discursivo como algo meramente humano (Barad, 2003). De este modo, la investigación pasa a entenderse como un territorio en el que intra-actúan "en" y "dentro" los fenómenos materiales, discursivos, virtuales, humanos, no-humanos y más que humanos.

Desde estos principios, lo que en este artículo intentamos hacer, es poner en duda (para luego plantear preguntas) los supuestos ontológicos, epistemológicos, metodológicos y éticos que guían la investigación y lo que significa investigar, así como cuestionar nuestras propias investigaciones y posicionamientos que asumimos como investigadoras. Esto no significa que estemos proponiendo cambiar radicalmente nuestros supuestos ni el camino que inicialmente decidimos seguir en cada una de las tesis e investigaciones presentadas. Sin embargo, lo propuesto por esta perspectiva -y las/os autoras/es que en este texto se revisan-, nos cala profundamente y nos obliga a revisar algunas cuestiones que dábamos por sabidas y revisar cómo operamos continuamente dentro de la tradición que ha marcado la investigación en educación y en las ciencias sociales hasta ahora. Esto ha requerido asumir riesgos y transitar por caminos de no saber, poniendo a prueba nuestras propias concepciones sobre lo que significa investigar, y también la manera en que damos cuenta de ello.

De esta manera, dicha perspectiva alude a la multiplicidad y complejidad de nuestras experiencias y, a su vez, potencia una reflexión en ac- 
ción que nos invitan a pensar los procesos investigativos como enredos performáticos desafiando el "carácter neo-positivista de la investigación cualitativa" (Lather y St. Pierre, 2013). Desde aquí, nos proponemos explorar la perspectiva post-cualitativa como un espacio de reconceptualización de los procesos investigativos (y también educativos); es decir, un espacio que provee modos alternativos de pensar y producir nuestras investigaciones.

En las dos tesis que presentaremos a continuación se revisarán brevemente algunas categorías que se han impuesto para organizar y estructurar la metodología cualitativa humanista -por ejemplo, el problema de investigación, la(s) pregunta(s) de investigación como un punto de llegada, la recolección de datos, el análisis de datos, la representación, la noción de "evidencia", el rol del investigador/a dentro del proceso de entrevista y la "voz" del sujeto humanista-, las cuales asumen profundamente la idea de que el ser humano es superior "a" $y$ "separado de" lo material desde entendimientos binarios como yo/otros, sujeto/objeto, y humano/no humano (Lather y St. Pierre, 2013). Ambas tesis desde temáticas totalmente diferentes: una sobre el lugar del cuerpo en las trayectorias y formación inicial del profesorado de educación artística; y la otra, en torno al concepto de Proyecto Artístico desde la mirada de los artistas, la institución y el arte contemporáneo.

No obstante, una de las cuestiones principales que se ha puesto en tela de juicio sobre esta perspectiva es que, al abandonar el cientificismo positivista de las ciencias sociales, ya no podemos pensar en muchas de las categorías que hemos creído necesarias para garantizar el valor y el rigor de una investigación con enfoque cualitativo. En este sentido, Patti Lather y Elizabeth St. Pierre proponen una pregunta provocadora: "Without these, how do we know that what we are doing is science?" (2013, p. 630). Sin embargo, más allá de considerar esto un impedimento, lo entendemos como un desafío para pensar nuestras investigaciones fuera de los marcos establecidos.

Con todo, este artículo no pretende ser una invitación a "poner el contador a cero" (Hernández, 2015) sino a plantear cuestionamientos que generen desafíos y preguntas de una manera crítica frente a nuestros modos de hacer, entender y dar cuenta de la investigación. Es una invitación a estar abiertos a lo que nos cuestiona y amplía nuestros posicionamientos que, como es habitual, consideramos provisionales, móviles e inestables a fin de continuar aprendiendo. 
Asimismo, pone de manifiesto la necesidad de poner atención al lenguaje que utilizamos y a los conceptos/nociones a los que recurrimos continuamente para hablar de las personas y realidades con las que trabajamos, los métodos que seguimos y los procesos que entendemos como "correctos" al hacer investigación educativa, social y artística.

\section{Desplazamientos y giros disruptivos: ¿cómo la investigación se ha movido a partir de la perspectiva post-cualitativa?}

Uno de los capítulos que configuran mi tesis doctoral (Sara Carrasco), se titula "La conciencia del cambio". Creo que advertir de manera consciente los cambios que se pueden experimentar en la investigación durante su proceso de constitución y estar abierta a que ésta llegue a puertos inesperados, es algo fundamental dentro de una posición de investigadora que pretende ser nómade (Braidotti, 2004; Deleuze y Guattari, 1988) y llena a los devenires propios de los procesos investigativos, educativos y artísticos. Esta "conciencia" no solo significa aceptar los cambios que puedan suceder, sino también aceptar el error y entender que es posible aprender desde lo desconcertante, lo desconocido y lo imprevisto.

Comencé esta tesis queriendo abordar el problema inicial de investigación (centrado en la formación inicial del profesorado de artes visuales y el papel del cuerpo dentro de ella) desde una lógica bastante reductiva del cuerpo y de lo que éste significa dentro de la constitución del "ser docente". Esta lógica instintiva me llevó a entender el cuerpo desde su condición como construcción social mediante la cual intervienen fuerzas únicamente humanas.

En este recorrido inicial desarrollé una estrategia metodológica de investigación que fuese acorde con mis objetivos primeros y el marco teórico de la tesis. Para ello, realicé un estudio de casos colectivo (cinco mujeres y tres hombres entre veinte y veintisiete años) dentro del ámbito universitario público y estatal chileno. Me centré específicamente en las evidencias reunidas mediante las conversaciones y entrevistas semiestructuradas realizadas a los/as ocho profesores/as en formación. Por su parte, el análisis de esas historias se realizó tomando en cuenta algunos elementos y principios fundamentales propuestos por la segunda gene- 
ración de la Grounded Theory², desarrollada por autoras/es como Kathy Charmaz, Antony Bryant, Adele E. Clarke y Calder W. Kaufman, entre otros. Esto último, debido al rigor metodológico que aporta y al tratamiento profundo que quería darles a los relatos.

Sin embargo, cuando terminé el proceso de análisis, mi visión y posicionamientos frente a lo que pretendía investigar comenzó a cambiar. En esos momentos estaba viviendo una serie de cambios y devenires dentro de mi proceso formativo como investigadora y estaba ampliando mi mirada gracias a las herramientas conceptuales y de pensamiento que iba adquiriendo de lecturas y referentes post-cualitativos. Me di cuenta de que, al contrario de lo que pensaba, mi modo de acercarme a las historias de esos futuros profesores, aun habiendo realizado un proceso de análisis -en ocasiones bastante normativo-, se relacionaba más con la idea de expandir que de reducir datos. Tomar conciencia de esto, me ayudó a ver más allá de lo que quería y creía que iba a encontrar; me abrí a la posibilidad de considerar la emergencia de nuevos temas y conceptos que me permitían dialogar de otra manera con todo ello e ir más allá, para expandir el conocimiento que estaba construyendo.

Una de las cuestiones fundamentales de lo que significó este constante aprender desde el proceso de investigar, fue el de no renegar en ningún momento del camino recorrido, incluso, sabiendo que en ocasiones pudo haber sido bastante tradicional o positivista. Ese recorrido fue el que me ayudó a advertir y apreciar el giro ontológico, epistemológico y metodológico que estaba experimentado. Incluso, fue lo que me incentivó a narrar dicho giro en la escritura de la tesis como una manera de posicionarme éticamente frente a la investigación. Sin olvidar jamás lo que han puesto de manifiesto algunas autoras como Patti Lather, Maggie MacLure, Lisa A. Mazzei, Marg Sellers y Elizabeth St. Pierre, sobre "por qué miramos cómo miramos" y la influencia que la tradición tiene sobre esto, sobre la experiencia formativa de cada una de nosotras y sobre nuestras investigaciones. Nunca partimos de cero y por eso es necesario entender que la investigación es parte de ese itinerario.

Con esto en mente, comencé a pensar en la idea de hacer otro tipo de investigación y de ponerme en jaque como investigadora; hacer una investigación que solo diera cuenta de un proceso, ya no me bastaba. Mi intención era situarme desde una posición inestable para ver qué podía

2 Perspectiva construccionista de la Grounded Theory. 
emerger de allí, qué podía hacer con aquello de lo que estaba dando cuenta y cómo podía ponerlo en movimiento. Es decir, conectarme a la inestabilidad propia de investigar, del rebuscar (re-search) y de los conceptos que hacen parte de ella. En ese proceso se movió la investigación y yo dentro de ella como investigadora.

Este recorrido finalmente derivó en lo que es mi tesis doctoral hoy en día, "una construcción permanente que no solo se fundamenta en la interpretación de unos datos como resultado de una investigación, sino también, se fundamenta en la revisión y reinterpretación constante del recorrido de la misma experiencia de investigar" (Carrasco, 2017, p.35).

A continuación, explicaré brevemente los desplazamientos vividos a partir de tres momentos clave que revelan el giro ontológico y epistemológico de la investigación, descubriendo el cambio de mi mirada frente a lo que estaba estudiando y sobre el conocimiento que estaba construyendo.

\section{Cómo la investigación se ha movido en relación con el foco y la pregunta de investigación}

Uno de los giros importantes que tuvo la investigación fue decidir que la tesis no se centraría en el cómo se daba respuesta a la pregunta de investigación ${ }^{3}$, sino en poner la atención en lo que emergía -lo esperado y lo inesperado- de las historias de los participantes a partir de esa pregunta. Así como también, poner el foco en cómo yo podía dialogar con todo ello, poniendo atención a los conceptos que de ahí se desprendían.

Esto me llevó a repensar y reconceptualizar el proceso de escritura. El inicio de la tesis se abordó desde los desplazamientos vividos y no desde el problema inicial, a fin de darle un sentido más rizomático que lineal al proceso investigativo. Un proceso que intentó ensamblar e interconectar los múltiples caminos experimentados en un constante devenir (Deleuze y Guattari, 1983).

Por ello, creo que el valor de esta tesis no radica en la pregunta inicial y en cómo se da respuesta a ella a lo largo de la investigación, sino en todo lo que surgió a partir de ahí. Especialmente, en los conceptos ines-

3 La pregunta inicial era: ¿cómo se están "construyendo" las corporeidades y acciones performáticas de los(as) futuros(as) profesores(as) de artes visuales dentro de su proceso formativo? 
perados sobre el cuerpo y el territorio que éste ocupa para el profesorado en su formación inicial. El dejar aparcada la idea de dar respuesta a la pregunta inicial y a los objetivos de una manera precipitada, me ayudó a dirigir la mirada hacia lo que realmente se estaba entendiendo sobre el cuerpo y no hacia lo que esperaba encontrar.

Una vez hecho este ejercicio, decidí ensamblar los conceptos emergentes para construir una noción de cuerpo diferente a partir de lo que los(as) participantes decían. De aquí surgió un sentido más amplio del cuerpo que se extendía más allá de su atribución como construcción social y de un entendimiento binario de éste.

\section{Cómo la investigación se ha movido en relación con la metodología como modo de observar la realidad}

Entiendo la metodología como un modo de observar la realidad de manera reflexiva. En este sentido, dicha metodología se desplazó bajo un concepto: el movimiento. La investigación es algo que transita, que se mueve y que va de un lugar a otro sin emplazamiento fijo. Esta agitación constante fue causada, principalmente, por la mirada post-cualitativa y la revisión de algunos supuestos positivistas y fenomenológicos que se estaban dando por "correctos" en el proceso de investigación. En este sentido, la perspectiva post-cualitativa me ayudó a cuestionar algunos límites que estaba fijando sobre el tema explorado, para pensarlo luego en términos de simultaneidad y abandonar el sentido lineal inicialmente cedido.

Asimismo, muchos de los conceptos propios de la investigación cualitativa que estaba utilizando y entendiendo como correctos dentro de mi investigación, comencé a repensarlos desde lecturas post-humanistas y post-cualitativas muy significativas de autoras como Barad (2003), Lather (2013, 2016), MacLure (2013), Mazzei (2013), St. Pierre (2014), entre otras.

Específicamente, el texto $A$ voice without organs: interviewing in post humanist research de Lisa Mazzei (2013), me ayudó a entender y a reconceptualizar la noción de entrevista a partir de la relación cuerpo/materia/humano/no humano/más que humano. Esta autora crea un concepto llamado "Voz sin órganos" (Voice Without Organs) a partir de Deleuze y Guattari (Body Without Organs, 1983) para repensar tanto la entrevista como las voces que intentamos recoger. Mazzei propone entender la rela- 
ción entre investigador(a)-datos-participantes-teoría-análisis como una articulación de carácter performativo (a modo de enactment) donde ninguno de los elementos presentes es más valioso que el otro, ya que no pueden pensarse o existir sin la presencia del otro. Aquí, el enmarque o contexto es una parte inherente a esta relación donde tanto lo humano como lo material están presentes actuando en un mismo plano de relaciones.

De este modo, las entrevistas comenzaron a actuar como espacios de apertura e intra-acciones frente a lo que me iba sorprendiendo y a lo que no estaba previsto, intentando dialogar con los conceptos que emergían de las historias de los/as participantes y cómo éstos se movían en relación con los diferentes temas y sentidos del cuerpo dentro de la educación artística y la formación docente. Todo ello se trató de ensamblar a fin de generar una red de conexiones.

\section{Cómo la investigación se ha movido en relación con el sentido de cuerpo/corporeidad}

Desde estos territorios "post" logré pensar el cuerpo como una experiencia que se abre a un sin número de conexiones con potencias de todo tipo y que atraviesa el espectro social, educativo y político de los/as profesores/as en formación con quienes trabajé. El cuerpo ahora deviene experiencia circular, que transita y actúa de manera multidireccional e intensa pues está en conjugación con el mundo que los rodea; por tanto, se expande por todas las esferas del proceso formativo. Asimismo, la materia comienza a adquirir gran importancia (Matter comes to matter, Barad, 2003) en ese proceso.

Este entendimiento del cuerpo ya no se refiere a su orden fisiológico o biológico, ni tampoco al simbólico y cultural (corporalidad), entendido únicamente desde una atribución social resultante de acciones exclusivamente humanas, que se extendiende más allá de los límites físicos como parte constitutivamente subjetiva del ser humano. Éste es ahora un cuerpo que deviene experiencia performática y que se constituye por un entrelazamiento de fuerzas humanas, no humanas y materiales. Un entendimiento que pone en discusión los conceptos cuerpo, corporalidad y corpor(e)idad (Grosz, 1994, 2004). Este último, propuesto por la autora Elizabeth Grosz, reúne las zonas de contacto entre el cuerpo y el mundo, trasladando al cuerpo a un lugar inhumano, más que humano y más allá de lo humano. 
Con todo, tirar de hilos post-cualitativos, post-humanistas y los nuevos materialismos me ha ayudado a pensar el cuerpo desde otros territorios; como un todo que coexiste "con" y "en" múltiples dimensiones a la vez, un enredo (entanglement) compuesto por diversas fuerzas que actúan en un mismo plano de relación y que se enredan para producir algo distinto a lo que producirían de manera singular (Barad, 2012).

\section{Problematizar una investigación desde lo post-cualitativo: cómo tres sacudidas afectaron un viaje para redefinirlo}

Cuando pienso (Paola Villanueva) en lo post-cualitativo pienso en transformación, en vaivén, en apertura, en mestizaje, en devenir, en fragmentación, en caos, en conexión. Me imagino bebiendo un batido rico en combinaciones, capas y aromas, perdiéndome en un sabor complejo en el que, al final, todo encaja. Sin embargo, cuando empecé mi tesis doctoral no estaba tan cómoda con la idea de perderme en una tarea de estas características y, ni mucho menos, con la posibilidad de disfrutar en esta pérdida y de considerarla una fuente única de aprendizaje (Lather, 2007).

Planteé mi tesis -"De qué hablamos cuando hablamos de Proyecto Artístico. Derivas desde los artistas, la institución y el arte contemporáneo" (Villanueva, 2017)- como una indagación parcial en torno a la noción de Proyecto Artístico. Ésta partía de la pregunta "¿cómo se configura la praxis de un Proyecto Artístico?", desde la que se abrieron cuestiones sobre la construcción de la subjetividad en el arte, los sistemas de producción artística o los marcos institucionales en los que se desarrolla. Para problematizar estos interrogantes, nos fijamos en la Sala d'Art Jove, un centro referencial en Barcelona, perteneciente a la Generalitat, que se organiza en base a una convocatoria pública anual para creadores, investigadores, educadores, comisarios o, en definitiva, mediadores "emergentes" que trabajan en relación con el arte contemporáneo. La Sala nos abrió sus puertas y seis de los artistas seleccionados en la convocatoria del 2014 aceptaron implicarse en la investigación. A partir de la voz de estos artistas -Daniel, Harley, Irene, Joan, Marta y Pablo- construimos una narrativa fragmentada que pensaba el Proyecto Artístico como un dispositivo artístico, social, económico, político y/o pedagógico, a la vez que visibilizaba cómo investigar a la deriva. Hoy, 
un año más tarde, reconozco esta tesis como el espacio en el que empecé a investigar post-cualitativamente (o en el que traté de hacerlo).

\section{Sacudida 1}

Lo post-cualitativo se cruzó con mi camino en la recta final de la tesis, lo cual explica la perturbación que esto supuso en tanto que replanteamiento de un trabajo que, en esa fase, debía ser sólido. Autoras como Patti Lather, Elizabeth Adams St. Pierre, Lisa A. Mazzei o Maggie MacLure fueron clave en este replanteamiento.

Por aquel entonces, antes de cruzarme con estas autoras, reposaba sobre el marco epistemológico y ontológico que me había abierto el construccionismo social, mediante el cual había desarrollado una mirada desconfiada sobre lo que había venido considerando como natural, correcto o evidente (íñiguez, 2003). En lo que se refiere al sentido de investigar, esta desconfianza partía de un rechazo a ideas como "objetividad" y "Verdad" que, bajo ciertos preceptos positivistas que pueden orientar una investigación hasta definirla, antes asociaba al rigor académico y a lo que una investigación "debía ofrecer" (Ibáñez, 2001). Desde el construccionismo social, me abracé a la posibilidad de investigar teniendo en cuenta la complejidad en la que sucede una investigación, entendiéndola como una de las posibles realidades resultantes, versionadas, subjetivizadas, relatadas y construidas en la relación de contextos, sujetos y objetos particulares (Gergen, 1996).

Con estos antecedentes, las resonancias derivadas de mi aproximación a una perspectiva post-cualitativa -desde textos de Jackson y Mazzei (2012), Lather (2013), Mazzei (2013) y St. Pierre (2014)- no me resultaban tan distintas a las que había ido recogiendo en el máster Arts visuals i educació: un enfocament construccionista y los posteriores estudios de doctorado en la Universitat de Barcelona. No obstante, lo que estos textos planteaban me parecía nuevo en lo que se refiere a cómo investigar y cómo dar cuenta de ello, poniendo en duda los cimientos sobre los que había edificado mi trabajo y toma de decisiones. Me pregunté, entre otras cuestiones, de qué manera había activado las metodologías cualitativas de investigación seleccionadas en las primeras etapas de ésta, si me estaba limitando a reducir experiencias en forma de "datos" o "evidencias", si estaba anteponiendo el "saber" sobre el "ser", o si estaba instrumentalizando a las personas con las que había trabajado 
para dar respuesta a mis hipótesis. En relación con ello, leyendo a St. Pierre (2014), me pregunté si estaba "reproduciendo unos modelos que canonizaban la estructura de una investigación cualitativa que corría el riesgo de convertirse en una nueva forma de positivismo o humanismo ilustrado" (Villanueva, 2017, p. 34).

\section{Sacudida 2}

En la tesis, las entrevistas tenían un papel protagonista, ya que en estos espacios se estaba construyendo la investigación. De ahí que repensar la concepción y el sentido de éstas desde un posicionamiento post-cualitativo fuera fundamental para resituarlas en este enmarcado -entendido como un proceso situado en el espacio y el tiempo-, en el que se apuesta por la movilidad, la transformación o lo transversal (Bal, 2009).

Cuando me topé con la perspectiva post-cualitativa, venía de una noción de entrevista abierta o semiestructurada que había ubicado en lecturas seminales sobre la etnografía y sus métodos de investigación (Hammersley y Atkinson, 2001). Pero, sin rechazarlas del todo, la lectura de Mazzei (2013) me llevó a repensar su función, su concepción y, sobre todo, cómo se genera el conocimiento en estos espacios. Pensar en las entrevistas como conversaciones compuestas por "voces sin órganos" que se desligan de un cuerpo específico -para producirse en la intersección entre la investigación, los datos, los participantes, la teoría y el análisis- me resultó reveladora. Lejos de buscar respuestas cuantificables o codificables, y cuestionando concepciones como "dato" o "evidencia", esta autora me llevó a pensar las conversaciones como espacios flexibles basados en la posicionalidad, la experiencia situada y/o la experiencia del self : "posicionalidad" entendida como los condicionantes y las relaciones en los que se construye el conocimiento (como el género, la posición social o la raza); "experiencia situada" como los conocimientos que están incorporados en los sujetos y sus experiencias vividas (Padró, 2011); y/o "experiencia del self", rememorando la idea feminista de que "lo personal es político", como detonante para prestar atención a la construcción de mi propia subjetividad como mujer, blanca y de clase media en el proceso de investigación.

Concebir las conversaciones de esta manera me llevó a vivirlas como "acontecimientos pedagógicos" (Atkinson, 2012) en sí mismos, como lugares de producción de aprendizajes significativos. Pero también, como 
lugares de quiebre, ruptura y disrupción de los cuales emergían conocimientos encarnados (Haraway, 1988), y cuya codificación tenía poco o ningún sentido desde una perspectiva post-cualitativa. De haberlo hecho, ¿habría caído en una estatización del conocimiento?, ¿en una especie de experiencia cuantificable y objetivada?, ¿en una nueva forma de positivismo o en un método prescriptivo y excesivamente planeado, como decía St. Pierre (2014)?

\section{Sacudida 3}

Pese a haber empezado a escribir demasiado tarde -al pensar que la escritura se ubica al final de proceso de trabajo, una vez analizadas e interpretadas las "evidencias" recogidas en las conversaciones-, encontré en la escritura el hipocentro para performatizar un pensamiento postcualitativo y dar cuenta de la impronta que éste estaba dejando.

Habiendo incorporado, ya desde el inicio, el pensamiento nómade de Braidotti (2000, 2004), la escritura se presentó como el lugar idóneo para dar cuenta del tránsito, del devenir, de la transformación y de la pérdida. Es decir, como la oportunidad para visibilizar cómo había ido adaptándome y traduciendo las realidades y experiencias con las que me había relacionado durante el camino, y cómo había aprendido en esta transitoriedad. Fue así como decidí desligarme de un guion que supuestamente debía permitirme cerrar el contenido de la tesis antes de escribirla, y empecé a operar en base a la noción de plugging in. Esta noción, que Jackson y Mazzei (2012) plantean a partir del pensamiento de Deleuze y Guattari (1988), está directamente relacionada con la idea de proceso y, de esta manera, con un ejercicio de hacer y deshacer, con una apertura a organizar y desorganizar la escritura en base a conexiones múltiples y, a veces, inesperadas o irresueltas. En esta manera de entender la escritura como un "assemblage in formation" (Jackson y Mazzei, 2012), asumí el rol de investigadora bricoleur en el que, reposando sobre algunas claves del giro narrativo, pude visibilizar la fragmentación de mi yo-investigadora, me permití una escritura también fragmentada e inconclusa (como lo es a menudo el pensamiento), y presté atención, sin olvidar las descripciones escenográficas, a lo que ocurría entre las experiencias personales y los contextos sociales (Hernández y Rifà, 2011).

Al tomar conciencia de la escritura como un ejercicio de conexiones 
y desconexiones, me vi inmersa en el rizoma de Deleuze y Guattari (2010). En una forma de rizoanálisis que, lejos de funcionar como un método, configura una manera de investigar (o una manera de escribir) horizontal, impredecible, por capas, que puede parecer caótica y desorganizada, pero que permite mostrar cómo se construye y deconstruye la investigación, y al investigador en esta maraña (Masni, 2014).

\section{Otro punto y aparte}

Coherente a este posicionamiento, colocar un apartado final a la tesis parecía accesorio, casi un acto de desconfianza a mi propio trabajo. Pero, alertada por los estándares de lo que puede esperarse de una investigación en el campo académico (y en el marco de unos estudios de doctorado), me resultaba difícil obviar los riesgos que suponía alterar la estructura tradicional de una tesis. Fernando Hernández, director de la tesis y compañero de viaje, me animó a apostar por un no-final en forma de coda, en la cual respondería a la propuesta post-cualitativa de pensar otras maneras investigar. Como una forma de Proyecto Artístico -o también como una forma de mirar el arte contemporáneo desde su inmaterialidad, y desde los valores y significados simbólicos que se producen en ella (Sansi, 2015)- un no-final no quiere decir no tener conclusiones. Más bien, supone evidenciar cómo éstas, en forma de conocimientos a la deriva, se han expandido a lo largo de la narración, haciendo hincapié en el valor de lo procesual. Aún con el sabor de boca de aquel batido rico y complejo, me sigo recreando en la idea de que aquello que puede producirse al mirar y ensamblar la realidad desde sus pliegues, repliegues y despliegues (Deleuze, 2014), posibilita conocimientos únicos, poderosos e infinitos. Y adoptar un posicionamiento post-cualitativo puede ser un lugar, no siempre cómodo, para experimentarlo.

\section{Un espacio para seguir pensando en voz alta}

Al escribir un artículo sobre la perspectiva post-cualitativa como reconceptualización de la investigación, muchas cuestiones se ponen en juego. Ya lo decían Patti Lather y Elizabeth St. Pierre (2013): en primer lugar, reaparece el debate sobre si la investigación post-cualitativa se puede 
considerar investigación dentro de los marcos establecidos y el cientificismo positivista de las ciencias sociales; segundo, hacia dónde va esta perspectiva, ¿existen riesgos de que también llegue a convertirse en un método hegemónico y sistemático en forma de dogma metodológico?; tercero, al abandonar el cientificismo positivista de las ciencias sociales, ¿se está perdiendo con ello la garantía del valor y el rigor de nuestras investigaciones?

Si bien todas estas cuestiones expuestas son legítimas y apropiadas en cuanto el temor que genera partir de algo nuevo y desconocido, no dejan de centrar el debate en los aspectos negativos de la discusión y colocan ambos planteamientos en contra del otro de una manera dicotómica. Asimismo, no escapan de una crítica reductiva que finalmente terminará por dominar y constreñir estos intentos por construir y pensar una investigación otra. Sobre esto, Marina Garcés (2017) advierte lo siguiente:

(...) Hemos llevado la crítica de las disciplinas y la ideología humanista hasta tal punto que durante años las artes y las ciencias humanas han tendido a reducirse a ser una crítica de sí mismas y de sus presupuestos y efectos de dominación. Esto ha tenido como consecuencia que los estudios humanísticos hayan ido adoptando o bien una actitud defensiva o bien una actitud de constricción y de arrepentimiento. Ambas son poco interesantes y, en el fondo, paradójicamente cerradas y autorreferentes (pp. 67-68).

A partir de aquí irrumpen muchos otros interrogantes más, entre ellos: ¿hablamos de ruptura?, ¿de des-homogeneizar?, ¿de crear nuevas bases?, ¿Cómo se podría evitar caer, de nuevo, en una estandarización de las formas de hacer y pensar una investigación?, ¿es necesario fijar nuevos parámetros opuestos respecto a lo que se ha venido haciendo o del camino que se ha ido recorriendo?

Ya lo decíamos al inicio. Nuestra intención no es la de renegar de la tradición ni del trabajo realizado en nuestras investigaciones. Por eso, más que centrarnos en verbos como "borrar", "olvidar" o "sepultar" dentro de este debate, vemos que se nos presenta una vía hacia la apertura en la investigación social, educativa y artística. Para nosotras, el posicionarnos desde lo post-cualitativo para repensar nuestras investigaciones parte de la idea de "incorporar" y de "crear" nuevas posibilidades que no se basen en la negación, sino en la posibilidad de ser flexibles, to- 
mando aquellos elementos que nos permitan ser creativas en nuestras investigaciones con la humildad del que está queriendo hacer algo diferente y aprender de ello, desde rutas que pueden ser difusas y que, tal vez, sean vías potenciales para generar conocimientos y comunicarlos de otras maneras.

Por eso es por lo que también presentamos nuestras investigaciones de forma conjunta, porque a veces el camino puede ser solitario y repleto de tensiones y antagonismos. Tal vez busquemos la escucha, la recepción y el encuentro de los afectos que se ponen en juego al cuestionar nuestras propias investigaciones y posicionamientos asumidos como correctos durante años. Esto ha supuesto un esfuerzo significativo en cuanto a intentar otros modos de hacer, entender y dar cuenta de nuestras investigaciones; también ha requerido asumir riesgos y transitar por caminos de no saber, poniendo a prueba nuestras propias concepciones sobre lo que significa investigar -como profesoras, artistas e investigadoras- y teniendo en cuenta que la afectividad hace parte de la investigación y que el aprendizaje es un proceso que nos afecta (Camps, 2011; Clough, 2009; Domínguez y Lara, 2014; Massumi, 2011; Rivera de Rosales, 2011).

Más allá de las dificultades propias de esta experiencia, las contribuciones que nos deja la perspectiva post-cualitativa son vastas y evidentes en el proceso de repensar y reconfigurar un proceso de investigación y los supuestos ontológicos, epistemológicos, metodológicos y éticos que la guían, así como también, en torno a la discusión de algunas nociones y conceptos cualitativos humanistas y positivistas que han dejado de tener sentido para nosotras y que cobran un significado totalmente diferente desde su revisión.

La investigación post-cualitativa no es un campo que esté construido; se encuentra en construcción y, por ello, emergen numerosas tensiones. No obstante, lo importante para nosotras es no eludir esas tensiones; estas nos ayudan a enfrentarnos a lo que no sabemos y generar discusiones y debates a partir de ello. Finalmente, nos lleva a pensar sobre el modo de escritura que estamos utilizando y el uso del lenguaje (aunque construir una ontología desde el lenguaje tiene sus límites y sus riesgos porque puede dejar de lado la materialidad, como le ha pasado a otros enfoques investigativos). Con todos estos riesgos, decidimos seguir en esta exploración porque el hecho de escribir "desde" estas problemáticas y no "sobre" ellas, nos permite pensar sobre 
Movimientos y desplazamientos en la investigación. Lo post-cualitativo como reconceptualización en dos tesis doctorales

Sara Carrasco Segovia y Paola Villanueva González

aquello que estamos haciendo sin eludir las responsabilidades que ello conlleva.

\section{Referencias}

Atkinson, D. (2010). Looking Awry at the Notion of Core Competences in Visual Art Education. Journal of Research in Art Education, 12 (2), pp. 1-22. doi: 10.20977/ kkosea.2011.12.2.1

Atkinson, D. (2011). Art, Equality and Learning. Pedagogies Against the State. Rotterdam, Boston, Taipei: Sense Publishers.

Atkinson, D. (2012). Contemporary Art and Art Education: The New, Emancipation and Truth. International Journal of Art and Design Education, 31 (2), pp. 5-19.

Bal, M. (2009). Conceptos viajeros de las humanidades. Una guía de viaje. Murcia: Cendeac.

Barad, K. (2003). Posthumanist Performativity: Toward an Understanding of How Matter Comes to Matter. Journal of Women in Culture and Society 2003, 28 (3), pp. 801-831.

Barad, K. (2012). Thinking with intra-action. En A. Y. Jackson y L. A. Mazzei. (Eds.), Thinking with theory in qualitative research. Viewing data across multiple perspectives (pp. 118-136). New York: Routledge. i

Braidotti, R. (2000). Sujetos nómades. Buenos Aires: Paidós.

Braidotti, R. (2004). Feminismo, diferencia sexual y subjetividad nómade. Barcelona: Gedisa.

Camps, V. (2011). Spinoza: La fuerza de los afectos. En El gobierno de las emociones (pp. 65-87). Barcelona: Herder.

Carrasco, S. (2017). Desplazamientos y tránsitos en un proceso de investigación sobre el lugar del cuerpo en las trayectorias y formación inicial de profesores de educación artística (Tesis doctoral). Barcelona: Universidad de Barcelona.

Clough, P. (2009). The new empiricism. Affect and Sociological method. European Journal of Social Theory, 12 (1), pp.43-61.

Deleuze, G. (2014). El pliegue. Leibniz y el Barroco. Barcelona: Paidós.

Deleuze, G. y Guattari, F. (1983). Anti-Oedipus: Capitalism and schizophrenia. Minneapolis: University Press.

Deleuze, G. y Guattari, F. (1988). Mil mesetas. Capitalismo y esquizofrenia. Valencia: Pre-textos.

Deleuze, G. y Guattari, F. (2010). Rizoma (introducción). Valencia: Pre-textos.

Domínguez, E. y Lara, A. (2014). Emociones y ciencias sociales en el s. XX: La precuela del giro afectivo. Athenea Digital, 14(1), pp. 263-288. http://dx.doi.org/10.5565/rev/ athenead/v14n1.1094

Garcés, M. (2017). Nueva ilustración radical. Barcelona: Anagrama.

Gergen, K. (1996). Realidades y relaciones. Aproximaciones a la construcción social. Barcelona: Paidós. 
Movimientos y desplazamientos en la investigación. Lo post-cualitativo como reconceptualización en dos tesis doctorales

Sara Carrasco Segovia y Paola Villanueva González

Grosz, E. (1994). Volatile Bodies: Toward a Corporeal Feminism. Bloomington: Indiana University Press.

Grosz, E. (2004). The Nick of time: Politics, Evolution, and the Untimely. Durham: Duke University Press.

Hammersley, M. y Atkinson, P. (2001). Etnografía: métodos de investigación. Barcelona: Paidós.

Haraway, D. (1988). Situated Knowledges: The Science in Question in Feminism and the Privilege of Partial Perspective. Feminism Studies, 14 (3), pp. 575-599.

Hernández-Hernández, F. (2015). Introducción al seminario Investigación post-cualitativa. Programa de doctorado Artes y Educación. Barcelona: Universidad de Barcelona (no publicado).

Hernández, F. y Rifà, M. (Coords.) (2011). Investigación autobiográfica y cambio social. Barcelona: Octaedro.

Ibáñez, T. (2001). Municiones para disidentes. Realidad-Verdad-Política. Barcelona: Gedisa.

Íñiguez, L. (2003). La psicología social en la encrucijada postconstruccionista. Historicidad, subjetividad, performatividad, acción. XII Encontro Nacional da ABRAPSO. Estratégias de invenção - a Psicologia Social no contemporâneo. 15, 16 y 17 octubre del 2003, Pontifícia Universidade Católica do Rio Grande do Sul PUCRS. Puerto Alegre, Brasil. Recuperado el 4 de junio del 2012 de: http://abrapso. org.br/

Jackson, A. (2013). Posthumanist data analysis of mangling practices. International Journal of Qualitative Studies in Education, 26 (6), pp. 741-748.

Jackson, A. y Mazzei, L. (2012). Thinking with Theory in Qualitative Research. Viewing Data Across Multiple Perspectives. New York: Routledge.

Lather, P. (2007). Getting Lost: Feminist Efforts Toward a Double(d) Science. New York: State University of New York Press.

Lather, P. (2013). Methodology-21: What do we do in the afterword? International Journal of Qualitative Studies in Education, 26 (6), pp. 634-645.

Lather, P. (2016). (Re)Thinking Ontology in (Post)Qualitative Research. Cultural Studies/ Critical Methodologies, 16 (2), pp. 125-131.

Lather, P. y St. Pierre, E. (2013). Post qualitative research. International Journal of Qualitative Studies in Education, 26 (6), pp. 629-633.

MacLure, M. (2013). Researching without representation? Language and materiality in post-qualitative methodology. International Journal of Qualitative Studies in Education, 26 (6), pp. 658-667. doi: 10.1080/09518398.2013.788755

Masny, D. (2014). Disrupting Ethnography through Rhizoanalysis. Qualitative Research in Education, 3 (3), pp. 345-363. http://dx.doi.org/10.4471/qre.2014.51

Massumi, B. (2011). Palabras clave para el afecto. Exit Book: revista de libros de arte y cultura visual, 15, pp. 22-31.

Mazzei, L. (2013a). A voice without organs: interviewing in posthumanist research. International Journal of Qualitative Studies in Education, 26 (6), pp. 732-740. doi: 10.1080/09518398.2013.788761

Mazzei, L. (2013b). Materialist mappings of knowing in being researchers constituted 
Movimientos y desplazamientos en la investigación. Lo post-cualitativo como

reconceptualización en dos tesis doctorales

Sara Carrasco Segovia y Paola Villanueva González

in the production of knowledge. Gender and Education, 25 (6), pp. 776-785. doi: 10.1080/09540253.2013.824072

Padró, C. (2011). María Acaso. Elizabeth Ellsworth. El aprendizaje de lo inesperado. Madrid: Catarata.

Rivera de Rosales, J. (2011). Spinoza y los afectos. Exit Book: revista de libros de arte y cultura visual, 15, pp. 38-49.

Rogowska-Stangret, M. (2017). Corpor(e)al Cartographies of New Materialism: Meeting the Elsewhere Halfway. The Minnesota Review, 2017(88), pp. 59-68. doi: https://doi. org/10.1215/00265667-3787390

Sansi, R. (2015). Art, Anthropology and the Gift. London: Bloomsbury.

St. Pierre, E. (2014). A Brief and Personal History of Post Qualitative Research Toward Post Inquiry. Journal of Curriculum Theorizing, 30 (2), pp. 2-19.

Villanueva, P. (2017). De qué hablamos cuando hablamos de Proyecto Artístico. Derivas desde los artistas, la institución y el arte contemporáneo (Tesis doctoral). Barcelona: Universidad de Barcelona.

\section{Agradecimientos}

Esbrina - Subjetividades, visualidades y entornos educativos contemporáneos (2017SGR 1248).

REUNI+D -Red Universitaria de Investigación e Innovación Educativa. Cambios Sociales y Retos para la Educación en la Era Digital (EDU201568718-REDT). 